\title{
The value of the atherogenic index of plasma in non-obese people with non- alcoholic fatty liver disease: a secondary analysis based on a cross-sectional study
}

\author{
Bu-yuan Dong ${ }^{1 \dagger}$, Yu-qing $\mathrm{MaO}^{2 \dagger}$, Zheng-yang $\mathrm{Li}^{3^{*}}$ and Fu-jun Yu ${ }^{1^{*}}$ (D)
}

\begin{abstract}
Background and objectives: The atherogenic index of plasma (AIP) is elevated in fatty liver disease, but its value in non-obese people with non-alcoholic fatty liver disease (NAFLD) is unclear. This study aimed to investigate the relationship between AIP and NAFLD as well as to determine whether AIP might be used as an indicator of NAFLD in non-obese individuals.

Methods: The present study involved non-obese Chinese and Japanese participants. Risk factors are evaluated using univariate and multivariate analysis. The performance of risk factors was compared according to the area under the receiver operating characteristic curve.
\end{abstract}

Results: In the unadjusted model, the odds ratio (OR) for every 1 standard deviation (SD) increase in AIP was 52.30. In adjusted models I and II, the OR for every 1 SD increase in AIP was 36.57 and 50.84, respectively. The area under the receiver operating characteristic curve for AIP was 0.803 and 0.802 in the development and validation groups, respectively. The best cut-off value of AIP for discrimination between NAFLD and non-NAFLD was 0.005 in the Chinese group and -0.220 in the Japanese group.

Conclusions: AIP and NAFLD are positively correlated in Chinese and Japanese populations. Therefore, AIP can be used as a new screening indicator for non-obese people with NAFLD in different nations.

Keywords: Non-alcoholic fatty liver disease, Atherogenic index of plasma, Receiver operating characteristic curve, Non-obese patients, Odds ratio, Risk factor, Correlation

\section{Introduction}

Non-alcoholic fatty liver disease (NAFLD) is a common chronic liver disease worldwide. The disease may progress to liver cirrhosis and liver cancer [1,2]. Obesity is an important risk factor for NAFLD [3]. Liver histology

\footnotetext{
*Correspondence: noyes8002@126.com; tjyufujun@163.com

${ }^{\dagger}$ Bu-yuan Dong and Yu-qing Mao contributed equally to this work.

${ }^{3}$ Department of Gastroenterology, Jinshan Hospital of Fudan University, Jinshan, Shanghai 201508, China

'Department of Gastroenterology, the First Affiliated Hospital of Wenzhou Medical University, Nanbaixiang, Ouhai District, Wenzhou, Zhejiang, PR China Full list of author information is available at the end of the article
}

and non-invasive fibrosis tests suggest that some nonobese people may also have non-alcoholic steatohepatitis and advanced fibrosis despite having a body mass index (BMI) within the normal range [4-6].

The plasma atherosclerosis index (AIP) is a quantitative indicator used to evaluate blood lipid levels. AIP has good predictive value for dyslipidemia diseases such as diabetics, atherosclerosis and heart disease [7-9]. AIP has important predictive value not only for cardiovascular disease but also for hyperuricemia [10]. Several studies have reported a positive correlation between high levels of

C C The Author(s). 2020 Open Access This article is licensed under a Creative Commons Attribution 4.0 International License, which permits use, sharing, adaptation, distribution and reproduction in any medium or format, as long as you give appropriate credit to the original author(s) and the source, provide a link to the Creative Commons licence, and indicate if changes were made. The images or other third party material in this article are included in the article's Creative Commons licence, unless indicated otherwise in a credit line to the material. If material is not included in the article's Creative Commons licence and your intended use is not permitted by statutory regulation or exceeds the permitted use, you will need to obtain permission directly from the copyright holder. To view a copy of this licence, visit http://creativecommons.org/licenses/by/4.0/. The Creative Commons Public Domain Dedication waiver (http://creativecommons.org/publicdomain/zero/1.0/) applies to the data made available in this article, unless otherwise stated in a credit line to the data. 
AIP and obesity [11], and have shown that AIP has better discriminatory ability for NAFLD in obese people [12]. However, there has been little relevant research on nonobese people. More attention should be paid to non-obese people with NAFLD, who often think that a fatty liver is unlikely in the absence of obesity.

This study aimed to investigate the relationship between AIP and NAFLD in non-obese people and to demonstrate that AIP is an independent risk factor for NAFLD in non-obese individuals.

\section{Materials and methods}

\section{Data sources}

Data in the study came from the Dryad Digital Repository website (www.datadryad.org), which allows users to download raw data free of charge. These data are anonymous. According to the Dryad Terms of Service, researchers may apply these data in secondary analysis without infringing on the authors' rights. In the study, the Chinese data came from the following source [13]: Association of lowdensity lipoprotein cholesterol within the normal range and NAFLD in the non-obese Chinese population: a cross-sectional and longitudinal study. Dataset website: https://doi.org/10.5061/dryad.1n6c4. The Japanese data came from the following source [14]: Ectopic fat obesity presents the greatest risk for incident type 2 diabetes: a population-based longitudinal study. Dataset website: https://doi.org/10.5061/dryad.8q0p192. Variables included in the Chinese database file were as follows: age, sex, $\gamma$ glutamyltranspeptidase (GGT), alanine aminotransferase (ALT), aspartate aminotransferase (AST), total protein (TP), albumin (ALB), globulin (GLB), total bilirubin (TB), direct bilirubin (DBIL), blood urea nitrogen (BUN), creatinine $(\mathrm{Cr})$, estimated glomerular filtration rate (eGFR), uric acid (UA), low-density lipoprotein cholesterol (LDLc), fasting plasma glucose (FPG), high-density lipoprotein cholesterol (HDL-c), total cholesterol (TC), triglyceride (TG), BMI, AIP and fatty liver. Variables included in the Japanese database file were as follows: age, GGT, ALT, AST, HDL-c, BMI, AIP and sex.

\section{Study design and participants}

For the Chinese study population, participants took part in health examination at the First Affiliated Hospital of Wenzhou Medical University between January 2010 to December 2014. A total of 78,304 participants were recruited and selected on the basis of the following exclusion criteria: (1) a lack of required data; (2) excess alcohol consumption (more than $20 \mathrm{~g}$ per day for men or $10 \mathrm{~g}$ per day for women); (3) known liver disease; (4) $\mathrm{BMI} \geq 25 \mathrm{~kg} / \mathrm{m}^{2}$; (5) LDL-c $>3.12 \mathrm{mmol} / \mathrm{L}$; and (6) use of antihypertensive agents, antidiabetic agents or lipidlowing agents. Diagnosis of fatty liver was performed in accordance with the ultrasound diagnostic criteria of the
Chinese Liver Disease Association [15]. AIP was the base-10 logarithm of the ratio of the concentration of TG to HDL-c in concentration units of $\mathrm{mmol} / \mathrm{L}$, according to the formula AIP $=\log (\mathrm{TG} / \mathrm{HDL}-\mathrm{c})$ [16]. BMI was calculated as the weight in kilograms divided by the height in $\mathrm{m}^{2}$, and represented an index of body fat. All biochemical values were analyzed with an automatic measurement analyzer (Abbott) according to standard methods. Among the research population in Japan, a total of 12,932 participants who had undergone medical examination at Murakami Memorial Hospital between 2004 and 2015 were recruited and selected according to the following exclusion criteria: (1) a lack of important data; (2) known liver disease; (3) alcohol intake exceeding $60 \mathrm{~g}$ per day for men or $40 \mathrm{~g}$ per day for women; (4) drug use; (5) fasting blood glucose $\geq 6.1 \mathrm{mmol} / \mathrm{L}$; and (6) BMI $\geq 25 \mathrm{~kg} / \mathrm{m}^{2}$. Because this study was a secondary study, and the data were anonymous, no informed consent was required. Specific details are given in the original report [14].

\section{Diagnosis of NAFLD by ultrasonography}

NAFLD is defined by the diffusion enhancement of near-field echo and the gradual attenuation of far-field echo in the liver area (stronger than that in the kidney and spleen area). One of the following conditions must be present: (1) reduced blood flow signal but normal blood flow distribution; (2) mild to moderate hepatomegaly, with round and blunt borders;(3) unclear or incomplete envelope of the right liver lobe and the diaphragm muscle;(4) unclear liver cavity structure. NAFLD is diagnosed with abdominal ultrasonography performed by trained technicians.

\section{Statistical analysis}

The overall statistical analysis in this study consisted of five steps. First, in the Chinese study, the population was divided into a development group and validation group in a 7:3 ratio. Continuous variables are evaluated by calculating the means \pm standard deviations (SD) (normal distribution) or medians (quartiles) (skewed distribution), and categorical variables are evaluated by calculating frequencies or percentages. Differences across groups were analyzed using One-way ANOVA (normal distribution), Kruskal-Wallis $\mathrm{H}$ (skewed distribution) test and chisquare test (categorical variable). Second, risk factors in the development group were analyzed using univariate and multivariate regression analysis. Independent variables were tested for collinearity and were excluded with the variance inflation factor (VIF) $\geq 10$. Collinear VIF $=1 /(1$ $R^{2}$ ) [17]. The subgroups were grouped with a linear regression model. Third, according to the recommendation of the STROBE statement [18], the results of the unadjusted, minimally adjusted analysis and fully adjusted 
analysis are reported. Fourth, the area under the receiver operating characteristic (AUROC) curve of each predictor was used to compare the predictive utility. Fifth, box plots were used to intuitively reflect the predictive value of cutoff. All tests were two-sided. A $P$-value $<0.05$ was considered statistically significant. The $\mathrm{R}$ (version 3.4 .3 , The $\mathrm{R}$ Foundation; http://www.r-project.org) statistical package and GraphPad Prism (version 8.0; GraphPad Software) were used for statistical analysis.

\section{Results}

\section{Participants' baseline data}

As shown in Table 1, 78,304 Chinese participants were included in the study. The development group comprised 23,265 women and 31,608 men with an average age of 44.6 years. The average age, TP, ALB, GLB, TB, BUN, Cr, eGFR, UA, FPG, TC, LDL-c and BMI were greater in patients with than without NAFLD. The median GGT, ALT, AST, TG and AIP were greater in patients with than without NAFLD. The same trend was observed in the validation group. Among the Japanese participants, 11,598 people with NAFLD and 1334 people without NAFLD were included. The age, GGT, ALT, BMI, AIP, TG and FPG were greater in patients with than without NAFLD (Table S1).

\section{Univariate and multivariate analysis in the development group}

The univariate and multivariate analysis results are shown in Table 2. Univariate logistic regression results indicated that men were at higher risk of NAFLD than women. Higher age, GGT, ALT, AST, TP, ALB, GLB, TB, DBIL, BUN, Cr, UA, FPG, TC, TG, LDL-c, BMI and AIP were found in patients with than without NAFLD, thus indicating that these variables are risk factors in the progress of fatty liver disease. eGFR and HDL-c were lower in patients with than without NAFLD, thus indicating that these variables are protective factors. To exclude the mutual influence of these variables, variables with $\mathrm{VIF}<10$ were analyzed with a multivariate regression model. Age, GGT, ALT, ALB, DBIL, UA, FPG, LDL-c, BMI and AIP were independent factors positively

Table 1 Baseline Characteristics of the Chinese Study Participants

\begin{tabular}{|c|c|c|c|c|c|c|}
\hline \multirow[t]{2}{*}{ Characteristic } & \multicolumn{3}{|c|}{ Development Group } & \multicolumn{3}{|l|}{ Validation Group } \\
\hline & Non-NAFLD & NAFLD & $P$-value & Non-NAFLD & NAFLD & $P$-value \\
\hline No. of participants & 45,969 & 8904 & & 19,742 & 3689 & \\
\hline Age & $43.7 \pm 15.6$ & $49.4 \pm 13.5$ & $<0.001$ & $43.6 \pm 15.6$ & $49.7 \pm 13.8$ & $<0.001$ \\
\hline GGT (U/L) & $19.0(15.0,27.0)$ & $33.0(24.0,51.0)$ & $<0.001$ & $19.0(15.0,27.0)$ & $33.0(24.0,51.0)$ & $<0.001$ \\
\hline ALT (U/L) & $15.0(12.0,21.0)$ & $24.0(18.0,34.0)$ & $<0.001$ & $15.0(12.0,21.0)$ & $24.0(17.0,34.0)$ & $<0.001$ \\
\hline AST $(U / L)$ & $20.0(18.0,24.0)$ & $24.0(20.0,28.0)$ & $<0.001$ & $20.0(18.0,24.0)$ & $23.0(20.0,28.0)$ & $<0.001$ \\
\hline $\mathrm{TP}(\mathrm{U} / \mathrm{L})$ & $73.6 \pm 4.4$ & $74.3 \pm 4.3$ & $<0.001$ & $73.5 \pm 4.4$ & $74.3 \pm 4.4$ & $<0.001$ \\
\hline $\mathrm{ALB}(\mathrm{U} / \mathrm{L})$ & $44.5 \pm 2.8$ & $45.0 \pm 2.7$ & $<0.001$ & $44.5 \pm 2.8$ & $45.0 \pm 2.7$ & $<0.001$ \\
\hline GLB (U/L) & $29.0 \pm 4.0$ & $29.3 \pm 4.1$ & $<0.001$ & $29.0 \pm 3.9$ & $29.4 \pm 4.1$ & $<0.001$ \\
\hline $\mathrm{TB}(\mathrm{mmol} / \mathrm{L})$ & $12.5 \pm 5.0$ & $12.6 \pm 4.9$ & 0.012 & $12.5 \pm 5.2$ & $12.6 \pm 4.9$ & 0.004 \\
\hline DBIL (mmol/L) & $1.8(1.3,2.4)$ & $1.8(1.4,2.5)$ & $<0.001$ & $1.8(1.3,2.4)$ & $1.9(1.4,2.5)$ & $<0.001$ \\
\hline BUN (mmol/L) & $4.4 \pm 1.4$ & $4.7 \pm 1.3$ & $<0.001$ & $4.4 \pm 1.4$ & $4.7 \pm 1.4$ & $<0.001$ \\
\hline $\mathrm{Cr}(\mu \mathrm{mol} / \mathrm{L})$ & $81.2 \pm 24.2$ & $86.9 \pm 18.5$ & $<0.001$ & $81.3 \pm 25.5$ & $88.1 \pm 23.9$ & $<0.001$ \\
\hline eGFR (mL/min/1.73 m2) & $88.2 \pm 23.1$ & $88.0 \pm 22.9$ & $<0.001$ & $88.1 \pm 23.0$ & $87.9 \pm 23.1$ & $<0.001$ \\
\hline UA $(\mu \mathrm{mol} / \mathrm{L})$ & $278.5 \pm 85.7$ & $345.7 \pm 86.8$ & $<0.001$ & $279.1 \pm 85.6$ & $347.6 \pm 89.0$ & $<0.001$ \\
\hline $\mathrm{FPG}(\mathrm{mmol} / \mathrm{L})$ & $5.2 \pm 0.8$ & $5.7 \pm 1.4$ & $<0.001$ & $5.2 \pm 0.8$ & $5.7 \pm 1.3$ & $<0.001$ \\
\hline $\mathrm{TC}(\mathrm{mmol} / \mathrm{L})$ & $4.5 \pm 0.7$ & $4.8 \pm 0.8$ & $<0.001$ & $4.5 \pm 0.7$ & $4.8 \pm 0.8$ & $<0.001$ \\
\hline $\mathrm{TG}(\mathrm{mmol} / \mathrm{L})$ & $1.0(0.8,1.4)$ & $1.8(1.3,2.6)$ & $<0.001$ & $1.0(0.8,1.4)$ & $1.8(1.4,2.6)$ & $<0.001$ \\
\hline $\mathrm{HDL}-\mathrm{c}(\mathrm{mmol} / \mathrm{L})$ & $1.5 \pm 0.3$ & $1.2 \pm 0.3$ & $<0.001$ & $1.5 \pm 0.3$ & $1.2 \pm 0.3$ & $<0.001$ \\
\hline $\mathrm{LDL}-\mathrm{c}(\mathrm{mmol} / \mathrm{L})$ & $2.3 \pm 0.5$ & $2.4 \pm 0.5$ & $<0.001$ & $2.3 \pm 0.5$ & $2.4 \pm 0.5$ & $<0.001$ \\
\hline $\mathrm{BMI}\left(\mathrm{kg} / \mathrm{m}^{2}\right)$ & $21.3 \pm 2.1$ & $23.4 \pm 1.2$ & $<0.001$ & $21.3 \pm 2.0$ & $23.4 \pm 1.2$ & $<0.001$ \\
\hline AIP & $-0.1(-0.3,0.0)$ & $0.2(0.0,0.4)$ & $<0.001$ & $-0.1(-0.3,0.0)$ & $0.2(0.0,0.4)$ & $<0.001$ \\
\hline Sex & & & $<0.001$ & & & $<0.001$ \\
\hline Female & $21,650(47.1 \%)$ & 1615 (18.1\%) & & $9289(47.1 \%)$ & $654(17.7 \%)$ & \\
\hline Male & $24,319(52.9 \%)$ & 7289 (81.9\%) & & $10,453(52.9 \%)$ & 3035 (82.3\%) & \\
\hline
\end{tabular}


Table 2 Results of univariate and multivariate regression analysis in development group

\begin{tabular}{|c|c|c|c|c|c|c|}
\hline \multirow[t]{2}{*}{ Exposure } & \multicolumn{3}{|l|}{ Univariate } & \multicolumn{2}{|c|}{ Multivariate } & \multirow[b]{2}{*}{$P$-value } \\
\hline & $\mathrm{OR}$ & $(95 \% \mathrm{Cl})$ & $P$-value & $\mathrm{OR}$ & $(95 \% \mathrm{Cl})$ & \\
\hline \multicolumn{7}{|l|}{ Sex } \\
\hline Female & Reference & Reference & & Reference & & \\
\hline Male & 4.02 & $(3.80,4.25)$ & $<0.001$ & 0.77 & $(0.71,0.84)$ & $<0.001$ \\
\hline Age & 1.02 & $(1.02,1.02)$ & $<0.001$ & 1.01 & $(1.01,1.02)$ & $<0.001$ \\
\hline GGT (U/L) & 1.02 & $(1.02,1.02)$ & $<0.001$ & 1.00 & $(1.00,1.01)$ & $<0.001$ \\
\hline ALT $(U / L)$ & 1.04 & $(1.04,1.04)$ & $<0.001$ & 1.03 & $(1.03,1.03)$ & $<0.001$ \\
\hline AST (U/L) & 1.03 & $(1.03,1.04)$ & $<0.001$ & 0.97 & $(0.96,0.97)$ & $<0.001$ \\
\hline TP $(U / L)$ & 1.04 & $(1.03,1.05)$ & $<0.001$ & & & \\
\hline ALB $(U / L)$ & 1.07 & $(1.06,1.08)$ & $<0.001$ & 1.06 & $(1.05,1.07)$ & $<0.001$ \\
\hline GLB (U/L) & 1.01 & $(1.01,1.02)$ & $<0.001$ & 1.00 & $(0.99,1.01)$ & \\
\hline $\mathrm{TB}(\mathrm{mmol} / \mathrm{L})$ & 1.01 & $(1.00,1.01)$ & $<0.05$ & 0.99 & $(0.98,0.99)$ & $<0.001$ \\
\hline DBIL (mmol/L) & 1.07 & $(1.04,1.09)$ & $<0.001$ & 1.10 & $(1.07,1.14)$ & $<0.001$ \\
\hline BUN (mmol/L) & 1.13 & $(1.11,1.15)$ & $<0.001$ & 0.94 & $(0.92,0.96)$ & $<0.001$ \\
\hline $\mathrm{Cr}(\mu \mathrm{mol} / \mathrm{L})$ & 1.01 & $(1.01,1.01)$ & $<0.001$ & & & \\
\hline eGFR (mL/min/1.73 m2) & 0.99 & $(0.98,0.99)$ & $<0.001$ & & & \\
\hline UA $(\mu \mathrm{mol} / \mathrm{L})$ & 1.01 & $(1.01,1.01)$ & $<0.001$ & 1.00 & $(1.00,1.00)$ & $<0.001$ \\
\hline $\mathrm{FPG}(\mathrm{mmol} / \mathrm{L})$ & 1.55 & $(1.51,1.59)$ & $<0.001$ & 1.23 & $(1.20,1.26)$ & $<0.001$ \\
\hline $\mathrm{TC}(\mathrm{mmol} / \mathrm{L})$ & 1.70 & $(1.64,1.75)$ & $<0.001$ & 1.06 & $(0.99,1.14)$ & \\
\hline TG (mmol/L) & 2.62 & $(2.55,2.69)$ & $<0.001$ & 0.92 & $(0.88,0.96)$ & $<0.001$ \\
\hline $\mathrm{HDL}-\mathrm{c}(\mathrm{mmol} / \mathrm{L})$ & 0.11 & $(0.10,0.12)$ & $<0.001$ & & & \\
\hline LDL-c (mmol/L) & 2.18 & $(2.07,2.29)$ & $<0.001$ & 1.29 & $(1.16,1.42)$ & $<0.001$ \\
\hline $\mathrm{BMI}\left(\mathrm{kg} / \mathrm{m}^{2}\right)$ & 2.06 & $(2.02,2.09)$ & $<0.001$ & 1.69 & $(1.65,1.72)$ & $<0.001$ \\
\hline AIP & 52.30 & $(47.64,57.41)$ & $<0.001$ & 15.65 & $(12.85,19.05)$ & $<0.001$ \\
\hline
\end{tabular}

correlated with the progress of fatty liver disease, among which AIP was the strongest factor.

\section{Independent effect of AIP on the incidence of NAFLD} As shown in Table 3, the unadjusted odds ratio (OR) for every 1 SD increase in AIP was 52.30 (OR:52.30, 95\% CI, 47.64-57.41, $P<0.001)$. After adjustment for age, GGT, ALT, ALB, DBIL, UA, FPG, LDL-C and BMI (adjusted model I), the OR for every 1 SD increase in AIP was 36.57 (OR: 36.57, 95\% CI: 33.20-40.29; $P<0.001$ ). After full adjustment for sex, age, GGT, ALT, AST, TP, ALB, GLB, TB, DBIL, BUN, Cr, UA, FPG, TC, TG, LDL-C and BMI (adjusted model II), the OR for every 1 SD increase in AIP was 50.84 (OR: 50.84, 95\% CI: 38.22-67.63; $P<0.001)$. With OR $>1$ in three models, AIP was positively correlated with NAFLD, and the results were

Table 3 Effect modification of WHTR on incidence of NAFLD

\begin{tabular}{|c|c|c|c|c|c|c|}
\hline \multirow[t]{2}{*}{ Variable } & \multicolumn{2}{|l|}{ Unadjusted } & \multicolumn{2}{|l|}{ Adjusted Model I } & \multicolumn{2}{|l|}{ Adjusted Model II } \\
\hline & OR $(95 \% \mathrm{Cl})$ & $P$-value & OR $(95 \% \mathrm{Cl})$ & $P$-value & OR $(95 \% \mathrm{Cl})$ & $P$-value \\
\hline WHTR (Per SD) & $52.30(47.64-57.41)$ & $<0.001$ & $36.57(33.20-40.29)$ & $<0.001$ & $50.84(38.22-67.63)$ & $<0.001$ \\
\hline \multicolumn{7}{|l|}{ WHTR (quartile) } \\
\hline Q1 & Reference & & Reference & & Reference & \\
\hline Q2 & $3.18(2.78-3.63)$ & $<0.001$ & $2.67(2.34-3.05)$ & $<0.001$ & $1.80(1.56-2.08)$ & $<0.001$ \\
\hline Q3 & $7.91(6.98-8.96)$ & $<0.001$ & $5.88(5.18-6.68)$ & $<0.001$ & $2.74(2.37-3.18)$ & $<0.001$ \\
\hline Q4 & $28.67(25.41-32.35)$ & $<0.001$ & $19.64(17.36-22.23)$ & $<0.001$ & $5.04(4.26-5.95)$ & $<0.001$ \\
\hline$P$ for trend & $<0.001$ & & $<0.001$ & & $<0.001$ & \\
\hline
\end{tabular}

Model I adjusted for sex, age

Model II adjusted for sex, age, GGT, ALT, AST, TP, ALB, GLB, TB, DBIL, BUN, Cr, eGFR, UA, FPG, TC, TG, HDL-c, LDL-c, BMI $\mathrm{Cl}$ Confidence interval.SD $=0.29$ 
stable (Fig.S1). For further sensitivity analysis, AIP was converted to a categorical variable, and the results obtained were consistent (Table 3). Similar results were seen in the Japanese population (Table S2). Because AIP, age, GGT, ALT, ALB, eGFR, DBIL, UA, FPG, LDL-c and BMI were independent risk factors for NAFLD, their diagnostic performance for NAFLD were evaluated. AIP, which had the highest AUROC among these indicators, had the best discrimination capacity (AUROC: 0.803, 95\% CI: 0.798-0.808) in the development group (Fig.1 and Table 4), whereas DBIL had the worst performance (AUROC: $0.516,95 \%$ CI: 0.509-0.523). In the validation group, BMI performed the best (AUROC: $0.808,95 \% \mathrm{CI}$ : 0.801-0.814), and AIP ranked second (AUROC: 0.802, 95\% CI: 0.795-0.810). AIP had also the best discrimination ability (AUROC: $0.798,95 \%$ CI: 0.787-0.810) in the Japanese group (Fig.S2 and Table S3). Then the best cut-off value according to the maximum Youden index of the AUROC was determined. As shown in Fig.2, the best cut-off value of AIP in discriminating between NAFLD and non-NAFLD was 0.005 in the Chinese group and was -0.220 in the Japanese group.

\section{Subgroup analysis}

The subgroup analysis results are shown in Table 5 . The interactions were found to be significant for sex, age, AST, TB, BUN, UA, TC, TG, HDL-c, GGT, ALT, eGFR,
FPG and BMI $(P<0.01)$, whereas the tests for interactions were not statistically significant for ALB, GLB, DBIL and LDL-c $(P>0.05)$. Although all variables were risk factors, they did not affect the correlation between AIP and NAFLD. Compared with that of patients over 60 years old, the AIP in patients under 60 years old was associated with higher risks of NAFLD (OR: 61.03 VS. 25.67). Similar results were found in female and male patients (OR: 92.43 VS. 29.11).

\section{Discussion}

The purpose of this study was to analyze the relationship between AIP and NAFLD as well as to verify the diagnostic value of AIP in non-obese patients with NAFLD. AIP has been found to be an independent risk factor for NAFLD in non-obese patients through univariate and multivariate regression analysis, in agreement with results in obese patients [12]. Here, it was found that AIP was positively correlated with NAFLD in non-obese Chinese and Japanese patients. Subgroup analysis confirmed that many variables did not affect the positive correlation between AIP and NAFLD. Finally, AIP had better diagnostic value than the other variables in Chinese and Japanese patients, thus suggesting that AIP is applicable to different regions and ethnicities as a diagnostic indicator. However, the best cut-off value of AIP in discriminating between NAFLD and non-NAFLD

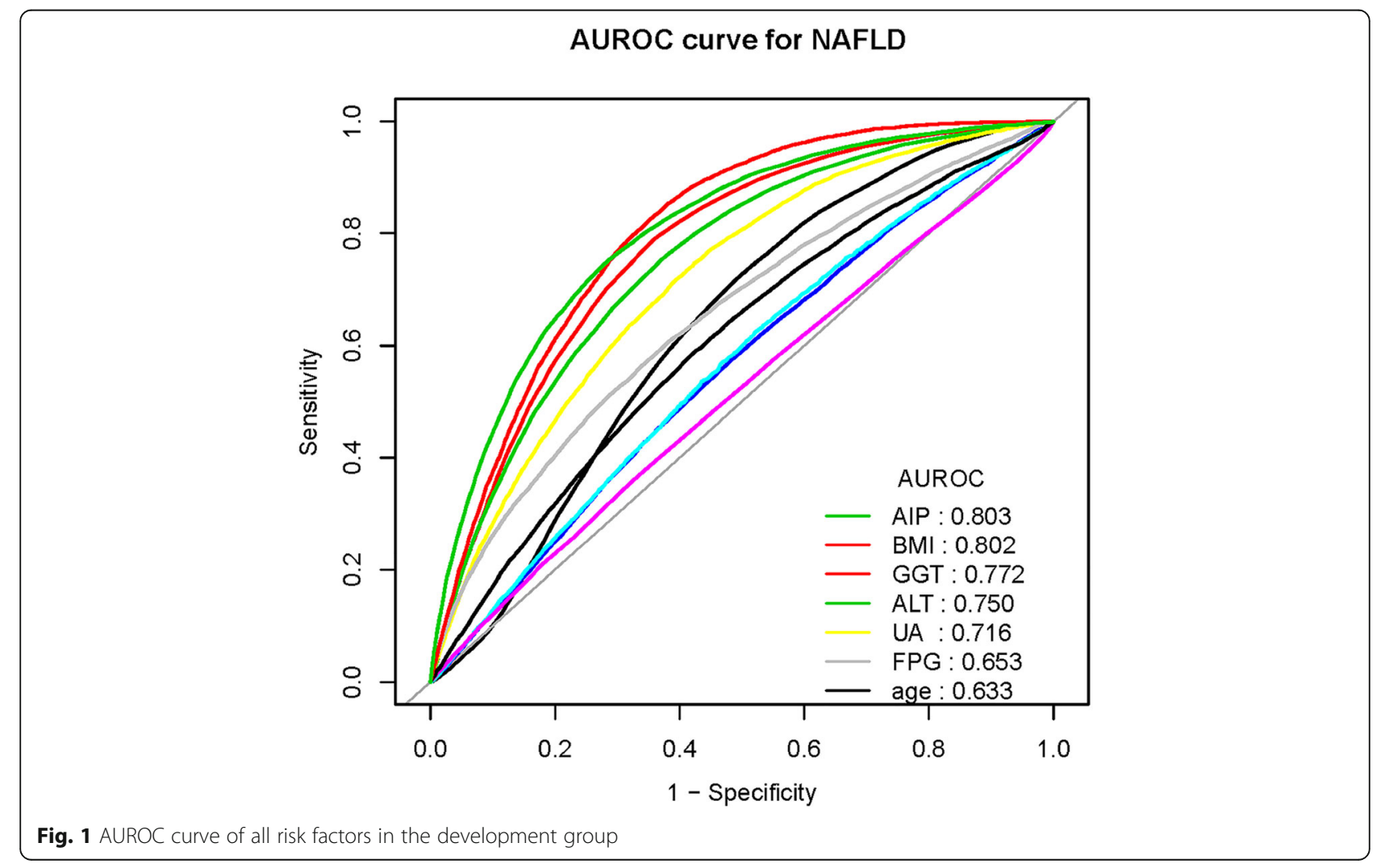


Table $4 \mathrm{ROC}$ analysis for different continuous predictors in development and validation groups

\begin{tabular}{|c|c|c|c|c|c|c|c|c|c|c|}
\hline & \multicolumn{5}{|c|}{ Development group } & \multicolumn{5}{|c|}{ Validation group } \\
\hline & AUROC & $95 \% \mathrm{Cl}$ & Cut-off pot & Specificity & Sensitivity & AUROC & $95 \% \mathrm{Cl}$ & Cut-off pot & Specificity & Sensitivity \\
\hline Age & 0.633 & $0.627-0.638$ & 40.500 & 0.511 & 0.717 & 0.637 & $0.629-0.646$ & 40.500 & 0.512 & 0.724 \\
\hline ALT & 0.750 & $0.745-0.756$ & 17.500 & 0.623 & 0.759 & 0.746 & $0.738-0.754$ & 17.500 & 0.626 & 0.743 \\
\hline GGT & 0.772 & $0.768-0.777$ & 22.500 & 0.639 & 0.792 & 0.772 & $0.765-0.780$ & 23.500 & 0.672 & 0.757 \\
\hline FPG & 0.653 & $0.646-0.659$ & 5.195 & 0.615 & 0.610 & 0.651 & $0.641-0.661$ & 5.225 & 0.640 & 0.578 \\
\hline ALB & 0.558 & $0.551-0.564$ & 44.650 & 0.517 & 0.573 & 0.556 & $0.546-0.566$ & 44.650 & 0.523 & 0.561 \\
\hline eGFR & 0.562 & $0.556-0.569$ & 67.165 & 0.479 & 0.625 & 0.577 & $0.567-0.587$ & 64.485 & 0.569 & 0.554 \\
\hline DBIL & 0.516 & $0.509-0.523$ & 2.150 & 0.676 & 0.359 & 0.530 & $0.519-0.540$ & 1.850 & 0.547 & 0.508 \\
\hline UA & 0.716 & $0.710-0.721$ & 295.500 & 0.609 & 0.716 & 0.717 & $0.708-0.725$ & 291.500 & 0.589 & 0.736 \\
\hline LDL-C, & 0.605 & $0.599-0.612$ & 2.375 & 0.5740 & 0.591 & 0.604 & $0.594-0.614$ & 2.385 & 0.584 & 0.579 \\
\hline BMI & 0.802 & $0.798-0.807$ & 22.335 & 0.647 & 0.826 & 0.808 & $0.801-0.814$ & 22.285 & 0.645 & 0.836 \\
\hline AIP & 0.803 & $0.798-0.808$ & 0.005 & 0.715 & 0.752 & 0.802 & $0.795-0.810$ & 0.025 & 0.735 & 0.733 \\
\hline
\end{tabular}

The unit is mmol/L: HDL-c, LDL-C, GGT, DBIL, FPG and UA; The unit is U/L: ALT and ALB

differed between Chinese and Japanese patients, thus demonstrating that AIP has different standards for different regions and ethnicities. This research provides a reference for future health examinations.

Previous studies have focused mainly on obese patients with BMI above 25, thus often causing people with normal BMI to ignore their eating habits [19]. Dietary habits may play a major role in the development of NAFLD in non-obese people. For example, excessive intake of saturated fat, fructose, sucrose and refined carbohydrates

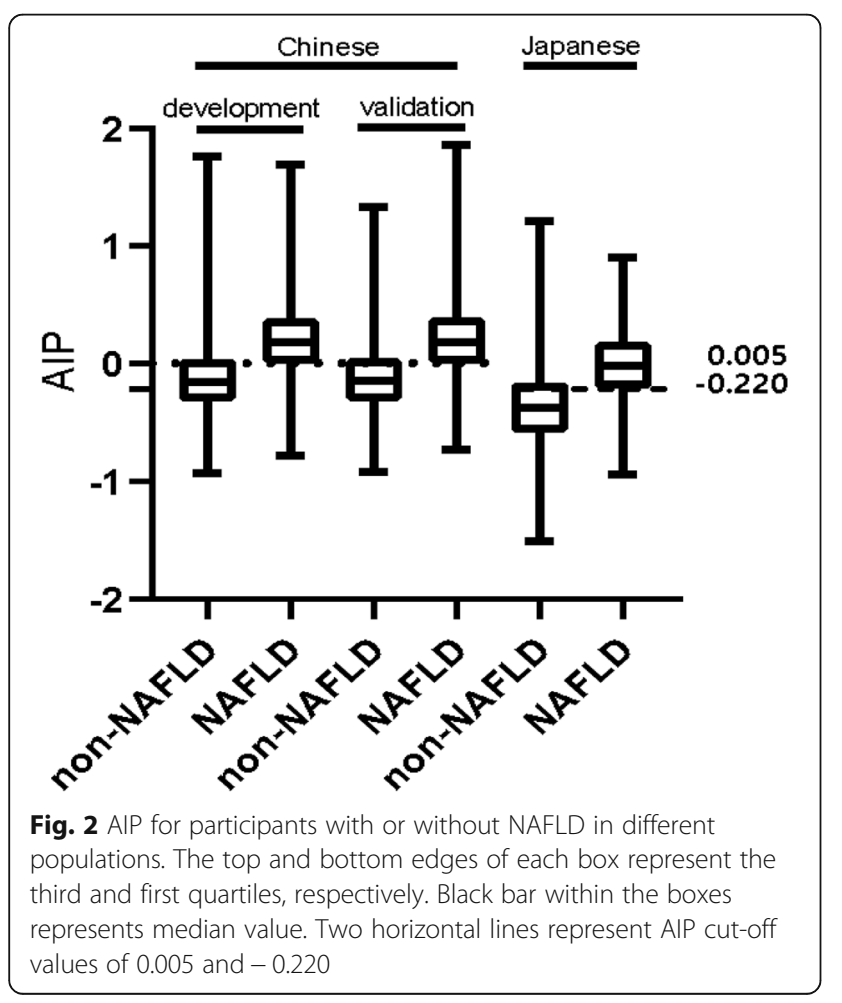

and low intake of n-3 polyunsaturated fatty acids, natural antioxidants (fruits and vegetables) and dietary fiber can promote the accumulation of triglycerides in the liver, thus increasing the prevalence of NFALD [20]. Therefore, These results may be alarming for people with BMI in a normal range. Because some studies have indicated that the prevalence of NAFLD in non-obese individuals varies greatly across regions [21, 22], this study included Chinese and Japanese patient populations to exclude regional disparities. The positive correlation between AIP and NAFLD may reflect the following findings: 1. Dysfunctional, expanded and inflamed adipose tissue may be associated with NAFLD in people with normal-range BMI [23]. AIP is part of traditional lipid profiles, but it is better than traditional pro-atherogenic lipid profiles [24]. AIP as an excellent combination factor may have good performance in the diagnosis of NAFLD. 2. Insulin resistance is an independent risk factor for NAFLD in non-obese people [25]. AIP is an independent predictor of insulin resistance [26-29]. Therefore, on the basis of previous studies and current findings, it is concluded that AIP is correlated with NAFLD. 3. NAFLD is characterized by the accumulation of triacylglycerol in the liver, in addition to increased oxidative stress and inflammation [30].

Previous research has indicated that TG/HDL-c can be used as predictor of NAFLD in non-obese people [31]. This study confirmed the previous findings and provides a new reference for clinical medicine. Through subgroup analysis, it was found that AIP and NAFLD are more closely associated in females than males, in agreement with previous results [31]. Unexpectedly, AIP was more closely associated with NAFLD in non-obese people under than over 60 years of age, a finding that has not been reported in the previous literature. This result may be associated with slower metabolism in older people. 
Table 5 Subgroup analysis of the association between AIP and NAFLD

\begin{tabular}{|c|c|c|c|c|c|}
\hline & $\mathrm{N}$ & OR & $95 \% \mathrm{Cl}$ & $P$ value & $P$ for interaction \\
\hline Sex & & & & & $<0.001$ \\
\hline Female & 23,265 & 92.43 & $75.30-113.45$ & $<0.001$ & \\
\hline Male & 31,608 & 29.11 & $26.10-32.46$ & $<0.001$ & \\
\hline Age (years) & & & & & $<0.001$ \\
\hline$<60$ & 45,394 & 61.03 & $54.95-67.78$ & $<0.001$ & \\
\hline$>=60$ & 9479 & 25.67 & $20.87-31.56$ & $<0.001$ & \\
\hline AST (U/L) & & & & & $<0.001$ \\
\hline$<21.500$ & 30,411 & 56.93 & $49.12-65.97$ & $<0.001$ & \\
\hline$>=21.500$ & 24,462 & 37.13 & $32.88-41.94$ & $<0.001$ & \\
\hline TB (mmol/L) & & & & & $<0.001$ \\
\hline$<9.750$ & 17,138 & 38.38 & $32.79-44.91$ & $<0.001$ & \\
\hline$>=9.750$ & 37,735 & 63.29 & $56.35-71.08$ & $<0.001$ & \\
\hline BUN (mmol/L) & & & & & $<0.001$ \\
\hline$<4.095$ & 23,047 & 78.67 & $66.96-92.43$ & $<0.001$ & \\
\hline$>=4.095$ & 31,826 & 40.22 & $35.87-45.09$ & $<0.001$ & \\
\hline UA (mmol/L) & & & & & $<0.001$ \\
\hline$<295.500$ & 30,509 & 68.38 & $57.90-80.76$ & $<0.001$ & \\
\hline$>=295.500$ & 24,364 & 25.37 & $22.55-28.55$ & $<0.001$ & \\
\hline $\mathrm{TC}(\mathrm{mmol} / \mathrm{L})$ & & & & & 0.001 \\
\hline$<4.525$ & 27,855 & 59.30 & $50.97-69.00$ & $<0.001$ & \\
\hline$>=4.525$ & 27,018 & 41.63 & $36.97-46.88$ & $<0.001$ & \\
\hline TG (mmol/L) & & & & & $<0.001$ \\
\hline$<1.395$ & 36,755 & 98.11 & $75.21-127.96$ & $<0.001$ & \\
\hline$>=1.395$ & 18,118 & 14.98 & $12.80-17.53$ & $<0.001$ & \\
\hline Total & 54,873 & 25.33 & 22.09-29.04 & $<0.001$ & \\
\hline $\mathrm{HDL}-\mathrm{c}(\mathrm{mmol} / \mathrm{L})$ & & & & & $<0.001$ \\
\hline$<1.335$ & 24,257 & 37.46 & $32.79-42.80$ & $<0.001$ & \\
\hline$>=1.335$ & 30,616 & 131.01 & 107.80-159.22 & $<0.001$ & \\
\hline GGT (U/L) & & & & & $<0.001$ \\
\hline$<22.5$ & 31,203 & 48.1 & $40.0-57.8$ & $<0.001$ & \\
\hline$>=22.5$ & 23,670 & 29.1 & $18.6-23.4$ & $<0.001$ & \\
\hline ALT (U/L) & & & & & $<0.001$ \\
\hline$<17.5$ & 30,801 & 47.1 & $39.9-55.6$ & $<0.001$ & \\
\hline$>=17.5$ & 24,072 & 28.7 & $25.5-32.2$ & $<0.001$ & \\
\hline ALB (U/L) & & & & & 0.656 \\
\hline$<44.65$ & 27,585 & 49.8 & $43.4-57.2$ & $<0.001$ & \\
\hline$>=44.65$ & 27,288 & 52.0 & $45.8-59.0$ & $<0.001$ & \\
\hline GLB (U/L) & & & & & 0.304 \\
\hline$<29.15$ & 28,786 & 54.8 & $48.1-62.5$ & $<0.001$ & \\
\hline$>=29.15$ & 26,087 & 49.7 & $43.5-56.8$ & $<0.001$ & \\
\hline DBIL (mmol/L) & & & & & 0.490 \\
\hline$<2.15$ & 36,777 & 55.4 & $49.4-62.1$ & $<0.001$ & \\
\hline$>=2.15$ & 18,096 & 51.6 & $43.9-60.7$ & $<0.001$ & \\
\hline eGFR & & & & & $<0.01$ \\
\hline
\end{tabular}


Table 5 Subgroup analysis of the association between AIP and NAFLD (Continued)

\begin{tabular}{|c|c|c|c|c|c|}
\hline & $\mathrm{N}$ & OR & $95 \% \mathrm{Cl}$ & $P$ value & $P$ for interaction \\
\hline$<67.165$ & 29,522 & 44.8 & $39.6-50.6$ & $<0.001$ & \\
\hline$>=67.165$ & 25,351 & 60.0 & $51.9-69.3$ & $<0.001$ & \\
\hline FPG (mmol/L) & & & & & $<0.001$ \\
\hline$<5.195$ & 31,735 & 61.8 & $53.6-71.2$ & $<0.001$ & \\
\hline$>=5.195$ & 23,138 & 35.8 & $31.6-40.6$ & $<0.001$ & \\
\hline LDL-c (mmol/L) & & & & & 0.678 \\
\hline$<2.375$ & 30,025 & 51.3 & $45.1-58.3$ & $<0.001$ & \\
\hline$>=2.375$ & 24,848 & 49.3 & $42.9-56.6$ & $<0.001$ & \\
\hline BMI (kg/m2) & & & & & $<0.001$ \\
\hline$<24$ & 46,508 & 51.2 & $45.8-57.3$ & $<0.001$ & \\
\hline$>=24$ & 8365 & 17.0 & $14.2-20.4$ & $<0.001$ & \\
\hline
\end{tabular}

However, the specific mechanism must be explored in the future.

\section{Study strengths and limitations}

The study has several strengths. First, this research was a large sample, multi-region and multi-ethnic study. Second, this was a retrospective study with many confounding factors, in which strict statistics was used to minimize residual confounding. Third, the authors identified effective cut-off points for AIP in Chinese and Japanese populations, thus providing data support for clinical diagnosis.

There were, however, several limitations to this study. First, because it was a secondary study, information on the lifestyles and eating habits of the participating population was not collected. Second, this study focused on the relationship between AIP and NAFLD, without further evaluation of other variables.

\section{Conclusion}

The results indicate that AIP and NAFLD are positively correlated in Chinese and Japanese populations. Therefore, AIP may be used as a new screening indicator for non-obese people with NAFLD in different nations, thus providing a reference for clinical work.

\section{Supplementary information}

Supplementary information accompanies this paper at https://doi.org/10. 1186/s12944-020-01319-2.

Additional file 1: Table S1. Baseline Characteristics of the Japanese Study Participants. Table S2. Effect of AIP on the incidence of NAFLD in Japanese. Table S3. AUROC analysis for different continuous predictors in Japanese groups. Figure S1. The relationship between AIP and NAFLD. Figure S2. AUROC curve of all risk factors in the Japanese group.

\section{Abbreviations}

GGT: Y-glutamyltranspeptidase; ALT: Alanine aminotransferase; AST: Aspartate aminotransferase; TP: Total protein; ALB: Albumin; GLB: Globulin; BUN: Blood urea nitrogen; TB: Total bilirubin; DBIL: Direct bilirubin; Cr: Creatinine;
eGFR: Estimated Glomerular Filtration Rate; LDL-c: Low-density lipoprotein cholesterol (LDL-c); UA: Uric acid; TG: Triglyceride; FPG: Fasting plasma glucose; TC: Total cholesterol; HDL-c: High-density lipoprotein cholesterol $(\mathrm{HDL}-\mathrm{C})$; AUROC: The area under the receiver operating characteristic curve; NAFLD: Non-alcoholic fatty liver disease; BMI: Body mass index; AIP: Atherogenic index of plasma

\section{Acknowledgements}

The authors thank Islam from Wenzhou Medical University for helping polish the article.

\section{Authors' contributions}

Buyuan Dong and Yuqing Mao designed the study. Buyuan Dong and Yuqing Mao extracted, collected and analyzed data. Buyuan Dong and Zhengyang Li prepared tables and figures. Buyuan Dong, Yuqing Mao, Zhengyang Li and Fujun Yu reviewed the results, interpreted data, and wrote the manuscript. All authors have made an intellectual contribution to the manuscript and approved the submission.

\section{Funding}

The project was supported by the National Natural Science Foundation of China (No. 81970527/H0317), Zhejiang Provincial Natural Science Foundation of China (No. LY19H030005), Wenzhou Municipal Science and technology Bureau (No. 2018Y0064).

\section{Availability of data and materials}

Data can be downloaded from the 'DATADRYAD' database (www.datadryad. org).

Ethics approval and consent to participate

This study was the second analysis of existing data. The data was

anonymous, so no informed consent is required.

\section{Consent for publication}

Not applicable.

Competing interests

The authors confirm that there are no conflicts of interest.

\section{Author details}

${ }^{1}$ Department of Gastroenterology, the First Affiliated Hospital of Wenzhou Medical University, Nanbaixiang, Ouhai District, Wenzhou, Zhejiang, PR China. ${ }^{2}$ Department of Gastroenterology, Shanghai First People's Hospital, School of Medicine, Shanghai Jiao Tong University, Shanghai, China. ${ }^{3}$ Department of Gastroenterology, Jinshan Hospital of Fudan University, Jinshan, Shanghai 201508, China. 
Received: 17 April 2020 Accepted: 10 June 2020

Published online: 23 June 2020

\section{References}

1. Brunt EM, Wong WW, Nobili V, Day CP, Sookoian S, Maher JJ, Bugianesi E, Sirlin CB, Neuschwander-Tetri BA, Rinella ME. Nonalcoholic fatty liver disease. Nat Rev Dis Primers. 2015;1:15080. https://doi.org/10.1038/nrdp. 2015.80 .

2. Fan J-G, Kim S-U, Wong WW-S. New trends on obesity and NAFLD in Asia. J Hepatol. 2017;67:862-73. https://doi.org/10.1016/j.jhep.2017.06.003.

3. Wong W, Wong GL, Yeung DK, Lau TK, Chan CK, Chim AM, Abrigo JM, Chan RS, Woo J, Tse YK, et al. Incidence of non-alcoholic fatty liver disease in Hong Kong: a population study with paired proton-magnetic resonance spectroscopy. J Hepatol. 2015;62:182-9. https://doi.org/10.1016/j.jhep.2014.08.041.

4. Wei JL, Leung JC, Loong TC, Wong GL, Yeung DK, Chan RS, Chan HL, Chim AM, Woo J, Chu WC, Wong WW. Prevalence and severity of nonalcoholic fatty liver disease in non-obese patients: a population study using protonmagnetic resonance spectroscopy. Am J Gastroenterol. 2015;110:1306-14; quiz 1315. https://doi.org/10.1038/ajg.2015.235.

5. Leung JC, Loong TC, Wei JL, Wong GL, Chan AW, Choi PC, Shu SS, Chim AM, Chan HL, Wong WW. Histological severity and clinical outcomes of nonalcoholic fatty liver disease in nonobese patients. Hepatology. 2017;65: 54-64. https://doi.org/10.1002/hep.28697.

6. Sookoian S, Pirola CJ. Systematic review with meta-analysis: the significance of histological disease severity in lean patients with nonalcoholic fatty liver disease. Aliment Pharmacol Ther. 2018;47:16-25. https://doi.org/10.1111/apt.14401.

7. Bora K, Pathak MS, Borah P, Hussain MI, Das D. Association of the Apolipoprotein A-I gene polymorphisms with cardiovascular disease risk factors and Atherogenic indices in patients from Assam, Northeast India. Balkan J Med Genet. 2017;20:59-70. https://doi.org/10.1515/bjmg-2017-0002.

8. Yang SH, Du Y, Li XL, Zhang Y, Li S, Xu RX, Zhu CG, Guo YL, Wu NQ, Qing P, et al. Triglyceride to high-density lipoprotein cholesterol ratio and cardiovascular events in diabetics with coronary artery disease. Am J Med Sci. 2017;354:117-24. https://doi.org/10.1016/j.amjms.2017.03.032.

9. Cai G, Shi G, Xue S, Lu W. The atherogenic index of plasma is a strong and independent predictor for coronary artery disease in the Chinese Han population. Medicine (Baltimore). 2017;96:e8058. https://doi.org/10.1097/MD. 0000000000008058.

10. Chang Y, Li Y, Guo X, Guo L, Sun Y. Atherogenic index of plasma predicts Hyperuricemia in rural population: a cross-sectional study from Northeast China. Int J Environ Res Public Health. 2016;13. https://doi.org/10.3390/ ijerph13090879.

11. Zhu X, Yu L, Zhou H, Ma Q, Zhou X, Lei T, Hu J, Xu W, Yi N, Lei S. Atherogenic index of plasma is a novel and better biomarker associated with obesity: a population-based cross-sectional study in China. Lipids Health Dis. 2018;17:37. https://doi.org/10.1186/s12944-018-0686-8.

12. Wang Q, Zheng D, Liu J, Fang L, Li Q. Atherogenic index of plasma is a novel predictor of non-alcoholic fatty liver disease in obese participants: a cross-sectional study. Lipids Health Dis. 2018;17:284. https://doi.org/10.1186/ s12944-018-0932-0.

13. Sun D-Q, Wu S-J, Liu W-Y, Wang L-R, Chen Y-R, Zhang D-C, Braddock M, Shi $\mathrm{K}-\mathrm{Q}$, Song $\mathrm{D}$, Zheng M-H. Association of low-density lipoprotein cholesterol within the normal range and NAFLD in the non-obese Chinese population: a cross-sectional and longitudinal study. BMJ Open. 2016;6:e013781. https:// doi.org/10.1136/bmjopen-2016-013781.

14. Okamura T, Hashimoto $Y$, Hamaguchi M, Obora A, Kojima T, Fukui M. Ectopic fat obesity presents the greatest risk for incident type 2 diabetes: a population-based longitudinal study. Int J Obes. 2019;43:139-48. https://doi. org/10.1038/s41366-018-0076-3.

15. Zeng MD, Fan JG, Lu LG, Li YM, Chen CW, Wang BY, Mao YM. Chinese National Consensus Workshop on nonalcoholic fatty liver D: guidelines for the diagnosis and treatment of nonalcoholic fatty liver diseases. J Dig Dis. 2008;9:108-12. https://doi.org/10.1111/j.1751-2980.2008.00331.X.

16. Wu TT, Gao Y, Zheng YY, Ma YT, Xie X. Atherogenic index of plasma (AIP): a novel predictive indicator for the coronary artery disease in postmenopausal women. Lipids Health Dis. 2018;17:197. https://doi.org/10. 1186/s12944-018-0828-z.

17. Vatcheva KP, Lee M, McCormick JB, Rahbar MH. Multicollinearity in regression analyses conducted in epidemiologic studies. Epidemiology (Sunnyvale). 2016;6. https://doi.org/10.4172/2161-1165.1000227.
18. Vandenbroucke JP, von Elm E, Altman DG, Gøtzsche PC, Mulrow CD, Pocock SJ, Poole C, Schlesselman JJ, Egger M, et al. Int J Surg (London, England). 2014;12:1500-24. https://doi.org/10.1016/j.ijsu.2014.07.014.

19. Araújo AR, Rosso N, Bedogni G, Tiribelli C, Bellentani S. Global epidemiology of non-alcoholic fatty liver disease/non-alcoholic steatohepatitis: what we need in the future. Liver Int. 2018;38:47-51. https://doi.org/10.1111/liv.13643.

20. Hernandez-Rodas MC, Valenzuela R, Videla LA. Relevant aspects of nutritional and dietary interventions in non-alcoholic fatty liver disease. Int J Mol Sci. 2015;16:25168-98. https://doi.org/10.3390/ijms161025168.

21. Younossi ZM, Koenig AB, Abdelatif D, Fazel Y, Henry L, Wymer M. Global epidemiology of nonalcoholic fatty liver disease-meta-analytic assessment of prevalence, incidence, and outcomes. Hepatology. 2016;64:73-84. https:// doi.org/10.1002/hep.28431.

22. Collaboration NCDRF. Trends in adult body-mass index in 200 countries from 1975 to 2014: a pooled analysis of 1698 population-based measurement studies with 19.2 million participants. Lancet. 2016;387:137796. https://doi.org/10.1016/S0140-6736(16)30054-X.

23. Sinn DH, Gwak GY, Park HN, Kim JE, Min YW, Kim KM, Kim YJ, Choi MS, Lee $J$ H, Koh KC, et al. Ultrasonographically detected non-alcoholic fatty liver disease is an independent predictor for identifying patients with insulin resistance in non-obese, non-diabetic middle-aged Asian adults. Am J Gastroenterol. 2012;107:561-7. https://doi.org/10.1038/ajg.2011.400.

24. Dobiasova M, Frohlich J. The plasma parameter log (TG/HDL-C) as an atherogenic index: correlation with lipoprotein particle size and esterification rate in apoB-lipoprotein-depleted plasma (FER (HDL)). Clin Biochem. 2001;34:583-8. https://doi.org/10.1016/s0009-9120(01)00263-6.

25. Armstrong Z, Wattenberg M. Visualizing statistical mix effects and Simpson's paradox. IEEE Trans Vis Comput Graph. 2014;20:2132-41. https://doi.org/10. 1109/TVCG.2014.2346297.

26. Song P, Xu L, Xu J, Zhang HQ, Yu CX, Guan QB, Zhao M, Zhang X. Atherogenic index of plasma is associated with body fat level in type 2 diabetes mellitus patients. Curr Vasc Pharmacol. 2018;16:589-95. https://doi. org/10.2174/1570161116666180103125456.

27. Yeh WC, Tsao YC, Li WC, Tzeng IS, Chen LS, Chen JY. Elevated triglycerideto-HDL cholesterol ratio is an indicator for insulin resistance in middle-aged and elderly Taiwanese population: a cross-sectional study. Lipids Health Dis. 2019;18:176. https://doi.org/10.1186/s12944-019-1123-3.

28. Pantoja-Torres B, Toro-Huamanchumo CJ, Urrunaga-Pastor D, GuarnizoPoma M, Lazaro-Alcantara H, Paico-Palacios S, Del Carmen R-SV, BenitesZapata VA, Insulin R. Metabolic syndrome research G: high triglycerides to $\mathrm{HDL}$-cholesterol ratio is associated with insulin resistance in normal-weight healthy adults. Diabetes Metab Syndr. 2019;13:382-8. https://doi.org/10. 1016/j.dsx.2018.10.006.

29. Pacifico L, Bonci E, Andreoli G, Romaggioli S, Di Miscio R, Lombardo CV, Chiesa C. Association of serum triglyceride-to-HDL cholesterol ratio with carotid artery intima-media thickness, insulin resistance and nonalcoholic fatty liver disease in children and adolescents. Nutr Metab Cardiovasc Dis. 2014;24:737-43. https://doi.org/10.1016/j.numecd.2014.01.010.

30. Valenzuela R, Videla LA. Impact of the co-administration of N-3 fatty acids and olive oil components in preclinical nonalcoholic fatty liver disease models: a mechanistic view. Nutrients. 2020;12. https://doi.org/10.3390/ nu12020499.

31. Chen Z, Qin H, Qiu S, Chen G, Chen Y. Correlation of triglyceride to highdensity lipoprotein cholesterol ratio with nonalcoholic fatty liver disease among the non-obese Chinese population with normal blood lipid levels: a retrospective cohort research. Lipids Health Dis. 2019;18:162. https://doi.org/ 10.1186/s12944-019-1104-6.

\section{Publisher's Note}

Springer Nature remains neutral with regard to jurisdictional claims in published maps and institutional affiliations. 